\title{
Toward a trace-free oxidant-insight into unexpected high yields of vapor phase polymerized polyterthiophene
}

\author{
Chun Ong ${ }^{1,4}$, Paul M Bayley ${ }^{2}$, Orawan Winther-Jensen ${ }^{3}$ and Bjorn Winther-Jensen ${ }^{1}$
}

Vapor phase polymerization (VPP) of conducting polymers has been widely reported as a versatile technique to obtain thin films with high conductivity and order. One oxidant commonly used for VPP is iron(III) para-toluenesulfonate (Fe(III)PTS) owing to its outstanding film-forming properties. The polymerization mechanism using Fe(III)PTS has been believed to follow the normal route, where (at least) two Fe(III) is needed for oxidizing one monomer unit to form the polymer. Surprisingly, when polymerizing terthiophene (TTh) monomers at $100^{\circ} \mathrm{C}$ using Fe(III)PTS, it was found that $\sim 2.3$ times more the expected amount of PTTh was produced, when long polymerization times were used. An explanation for the phenomenon was found when VPP of TTh was successful using only para-toluenesulfonic acid (PTSa) as oxidant. It is believed that PTSa is gradually converted to $\mathrm{SO}_{3}$ and toluene at elevated temperature and that reduction of $\mathrm{SO}_{3}$ is driving the oxidation of the TTh monomers. This finding also opens a useful oxidative polymerization route where the oxidant can be converted to volatile compounds leaving no trace in the end product.

Polymer Journal (2013) 45, 391-395; doi:10.1038/pj.2012.150; published online 8 August 2012

Keywords: conducting polymers; para-toluenesulfonic acid; polyterthiophene; vapor phase polymerization

\section{INTRODUCTION}

Intrinsically conducting polymers (ICPs) are currently being intensively researched, not only for their conductivity but also for their unique properties such as their ability to work in catalytic, electrochromic and light harvesting applications. ${ }^{1-6}$ Polythiophene is one ICP recognized for its high stability and tunable properties. By attaching different side groups to polythiophene, polymers with different band gaps and solubilities can be obtained, making it suitable for a wide range of applications. ${ }^{7-9}$

As many uses of ICPs, for example, electrodes require them as films, and most ICPs are hard or impossible to melt or dissolve after synthesis, ${ }^{2}$ significant research has focused on directly polymerizing smooth, ordered films of ICPs. Two well-known methods to do this are electrochemical polymerization and vapor phase chemical polymerization. ${ }^{10}$ However, electrochemical polymerization requires electrically conducting substrates, making this method unsuitable for coating of nonconducting surfaces. In addition, the production rate of this method is low and its cost is high. Vapor phase polymerization (VPP) is able to create smooth, highly conducting films without requiring a conducting substrate. ${ }^{11,12}$

During chemical polymerization of most ICPs, an oxidant is used to oxidize the monomer. For most common monomers, this requires removal of two hydrogen atoms from each monomer molecule. A mechanism for this reaction is shown in Figure $1^{13}$ for thiophene being oxidized using $\mathrm{Fe}(\mathrm{III}) \mathrm{Cl}_{3}$.

From Figure 1, it can be seen that $2 \mathrm{~mol}$ of $\mathrm{Fe}$ (III) oxidant are required to produce $1 \mathrm{~mol}$ of polymer repeat units. For terthiophene (TTh) the equation would be:

$$
n(\mathrm{TTh})+2 n \mathrm{Fe}^{3+} \rightarrow(\mathrm{TTh})_{n}+2 n \mathrm{H}^{+}+2 n \mathrm{Fe}^{2+}
$$

The 2:1 oxidant:monomer ratio has been experimentally confirmed in the case of VPP of pyrrole. ${ }^{12}$ The same ratio is expected for polymerization of other monomers proceeding by the same mechanism. This ratio has also been seen in electropolymerization and oxidative chemical polymerization, where at least $2 \mathrm{~mol}$ of electrons (oxidant) are required to produce $1 \mathrm{~mol}$ of polymer. ${ }^{10,13,14}$

In this report, measurement of the masses of iron(III) paratoluenesulfonate (Fe(III)PTS) oxidant used and polymer produced during chemical VPP of TTh showed that more polymer was produced than expected according to equation (1). In other words, the molar amount of polymer produced was more than half the molar amount of oxidant. Further, the difference of polymers made at different polymerization times were studied, which could indicate different mechanisms of polymerization over the course of the

\footnotetext{
${ }^{1}$ Department of Materials Engineering, Monash University, Clayton, Victoria, Australia; ${ }^{2}$ Institute for Technology Research and Innovation, Deakin University, Burwood, Victoria, Australia and ${ }^{3}$ School of Chemistry, Monash University, Clayton, Victoria, Australia

${ }^{4}$ Current address: Department of Materials Science and Engineering, National University of Singapore, Singapore, Singapore.

Correspondence: Dr B Winther-Jensen, Department of Materials Engineering, Monash University, Wellington Road, Clayton, Victoria 3800, Australia.

E-mail: bjorn.winther-jensen@monash.edu
}

Received 26 April 2012; revised 27 June 2012; accepted 7 July 2012; published online 8 August 2012 


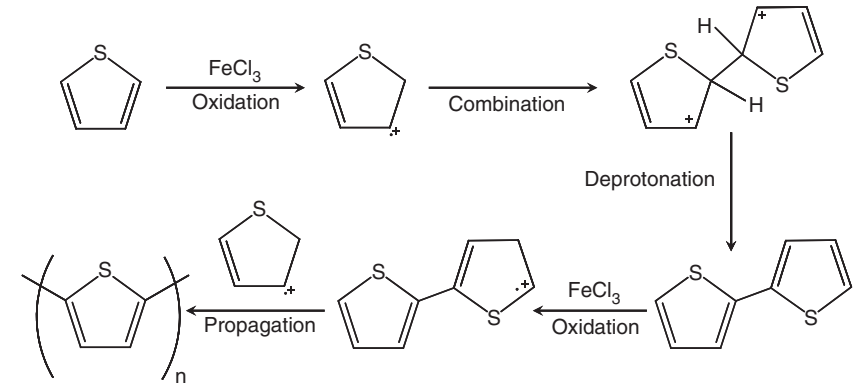

Figure 1 Chemical polymerization of thiophene using $\mathrm{FeCl}_{3}$ as oxidant. ${ }^{13}$

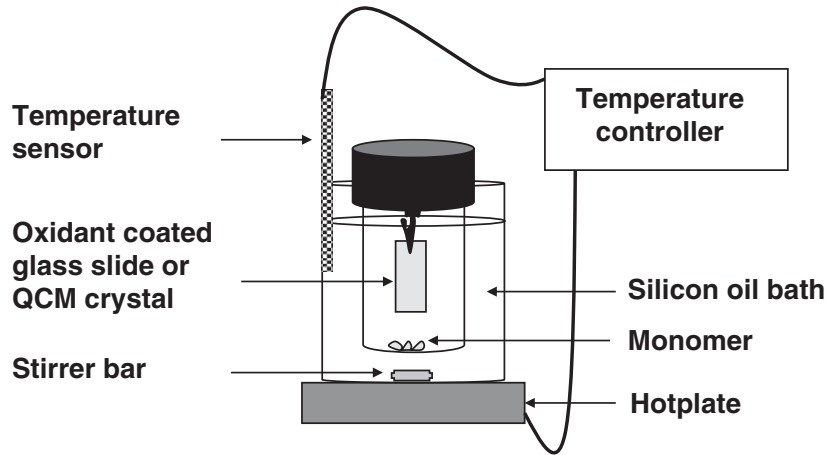

Figure 2 Schematic of VPP setup for TTh polymerization. A full color version of this figure is available at Polymer Journal online.

the laser and instrument is also used. All data are analyzed using the software package Grams AI 8.0 by Thermo Electron Corporation (Sydney, NSW, Australia).

Conductivity measurements were performed according to the procedure described previously. ${ }^{11}$ Two PEDOT films separated by an $\sim 6-\mathrm{mm}$ gap were polymerized on a single glass slide using VPP. PTTh was then polymerized to cover one of the PEDOT films and extend across the gap to within $\sim 2-3 \mathrm{~mm}$ of the other PEDOT film. The ionic liquid propyl methyl pyrrolidinium bis(trifluorosulfonamide) $\left(\mathrm{C} 3 \mathrm{mpyr}(\mathrm{Tf})_{2} \mathrm{~N}\right)$, synthesized in-house, was applied over the PTTh film, the gap and the exposed PEDOT film. The two-electrode cell was cycled between -1 and $+3 \mathrm{~V}$ twice and subsequently held at $3 \mathrm{~V}$ until the current leveled off after $\sim 3-5 \mathrm{~min}$. The applied potential was then removed, the cell disconnected and the film rinsed with ethanol to remove the ionic liquid, followed by drying under $\mathrm{N}_{2}$. Immediately, the surface conductivity was measured with a Jandel four-point probe (Jandel, Linslade, UK). The film thickness was analyzed using a DekTak profilometer (Veeco, Plainview, NY, USA) and the bulk conductivity was calculated.

\section{RESULTS AND DISCUSSION}

QCM results (Figure 3) showed that the expected amount of PTTh was produced after $\sim 2 \mathrm{~h}$ of polymerization. However, the amount of PTTh continued to increase until an amount about 2.3 times higher than expected was reached. To ensure the excess mass was polymer and not remaining oxidant or monomer, different samples were soaked for $1 \mathrm{~h}$ in ethanol, acetone or distilled water, and rinsed in butan-2-one, a good solvent for the monomer. This did not result in any significant change in mass. Samples were also dried over silica gel to remove moisture, which still did not change their mass.

Clearly, more polymer was produced than expected, up to $\sim 2.3$ times that expected according to equation (1), corresponding to a molar Fe(III):polymer(unit) ratio of 1:1.15. This 2.3-times limit did not change when using $140 \mu \mathrm{g}$ of oxidant (instead of $59 \mu \mathrm{g}$ ) on the QCM crystals or purging the polymerization chamber with nitrogen gas or air during polymerization. The fact that the 2.3 actual/expected limit is the same for different amounts of oxidant shows that the polymerization is stopping, and not just very slow.

This ratio of 1:1.15 seems almost magic and is not fitting with any polymerization mechanism for thiophenes found in the literature. If some kind of free-radical-like polymerization route was in play, it is not explainable why the reaction should stop at this (or any) welldefined ratio. The same argument can be used if regeneration of the oxidant by atmospheric oxygen was thought to be responsible and, as already mentioned, performing the polymerization under nitrogen or air did not change the result.

In order to further investigate the phenomenon, samples at different polymerization times were prepared and analyzed to clarify 


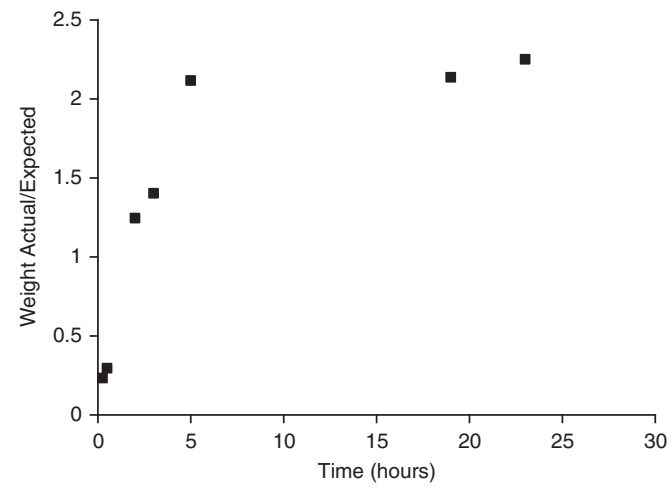

Figure 3 Mass of polymer produced over time after wash, as a multiple of expected mass according to equation (1), using $\sim 59 \pm 3 \mu \mathrm{g}$ of oxidant on the QCM crystal. Each point represents a separate experiment.

if there would be any change in the properties of PTTh produced with different polymerization times, that is, 3 and $16.5 \mathrm{~h}$. These times were chosen to produce two polymers corresponding to the time when $(3 \mathrm{~h})$ and long after $(16.5 \mathrm{~h})$ the Fe(III) oxidant was 'used up' according to equation (1).

Elemental analysis of the samples showed the ratio of C:H:S 4.3:3.1:1 and 4.2:2.6:1 for $3 \mathrm{~h}$ and $16.5 \mathrm{~h}$, respectively. The $\mathrm{C}$ to $\mathrm{S}$ ratio is thereby very close to the theoretical value (4:1) for both samples. The $\mathrm{H}$ content is somewhat higher for the shorter polymerization time, which indicates a lower average chain length. Conductivity of PTTh in its undoped state was very low and not possible to be measured using four-point probe technique. ${ }^{11}$ The conductivity values of the doped PTTh films were $17 \pm 1$ and $42 \pm 4 \mathrm{~S} \mathrm{~cm}^{-1}$ for $3 \mathrm{~h}$ and $16.5 \mathrm{~h}$, respectively, following the previously reported procedure. ${ }^{11}$ The conductivity for the 3-h samples is in very good agreement with earlier reports on VPP PTTh. ${ }^{11}$ However, the 16.5 -h samples showed higher conductivities, which may be assigned to slightly longer chain length as indicated from the elemental analysis.

The DSC scans of both samples show a peak within the range $130-140{ }^{\circ} \mathrm{C}$ (Figure 4). The DSC trace indicates a solid-solid phase transition, where the material is going from a more-ordered to lessordered state. ${ }^{11,16}$ The presence of this peak shows that the material is intrinsically ordered after polymerization. In the second scan, the peak disappeared, meaning the order was lost. These phenomena have been reported earlier for different $\mathrm{ICPs}^{11,16}$ and is also reported to reappear (reorder) after 3-5 days.

The sample polymerized for $16.5 \mathrm{~h}$ had a broader peak, with a lower transition energy of $63.67 \mathrm{Jg} \mathrm{g}^{-1}$, compared with $122.3 \mathrm{Jg}^{-1}$ for the 3-h sample. The width of the peak is believed to correspond to the distribution of ordered structures in the material, with a broader peak arising from a broader distribution. Having a lower transition energy means that the sample polymerized for $16.5 \mathrm{~h}$ has less order. It is an unexpected result to see that the 16.5-h samples have higher conductivity given that they possess a lower degree of order-this indicates that there is no direct correlation between high degree of $\pi$-stacking/order and conductivity for these samples. Taking the QCM results into account, the mass ratio between 3-h and 16.5-h samples should be about $1: 2$ and this could mean that most-ordered polymer in the 16.5-h sample was obtained during the first $3 \mathrm{~h}$ of polymerization. This change in the order obtained during the polymerization at and after $3 \mathrm{~h}$ indicates that there could indeed be a change in polymerization mechanism during the course of polymerization. It

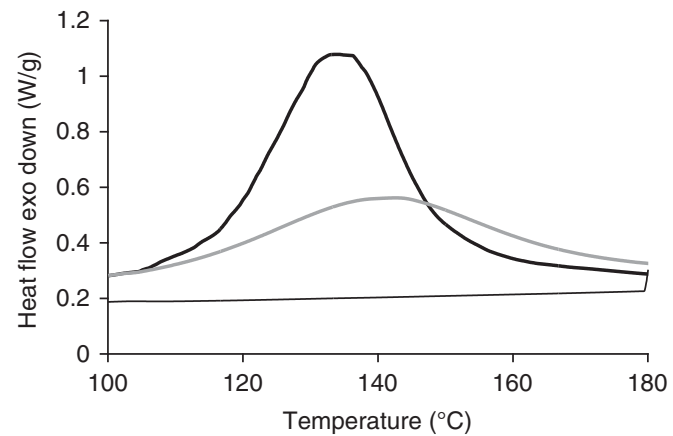

Figure 4 DSC scans of PTTh polymerized for 3 (thick black curve) and $16.5 \mathrm{~h}$ (gray curve). The thick black line represents the first scan, while the thin black line represents the second scan for the 3-h sample. Solid-solid transition energies were $122.3 \mathrm{~J} \mathrm{~g}^{-1}$ for the 3 -h sample and $63.67 \mathrm{Jg}^{-1}$ for the 16.5-h sample.

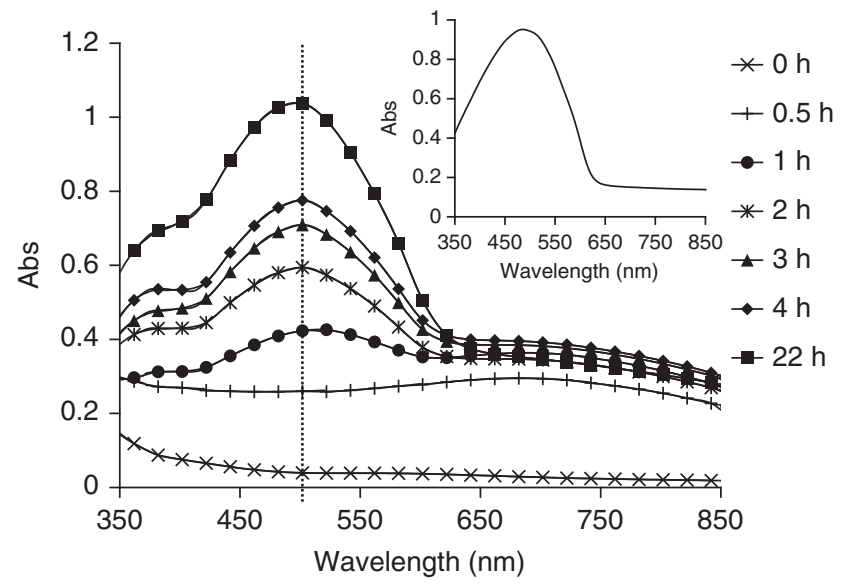

Figure 5 UV-vis spectrum of samples polymerized for various lengths of time without wash. Inset shows the spectrum of a sample polymerized for $22 \mathrm{~h}$ and washed in ethanol.

has previously been shown that the Fe(III) salt used for VPP has a templating influence on the order in PEDOT, ${ }^{16}$ resulting in significantly more order than in electropolymerized samples with the same cation. Imagining a shift in polymerization mechanism from a Fe(III)PTS templated to a non-templated route could result in the changes in DSC traces as seen for different polymerization times.

The UV-vis spectrum of unwashed samples during the polymerization in Figure 5 shows two peaks, at $\sim 500$ and $\sim 700 \mathrm{~nm}$ corresponding to the $\pi-\pi^{\star}$ transition and the polaron absorption and thereby to reduced and oxidized PTTh. These transitions also indicate the conjugation length of the conducting polymer. ${ }^{17}$ The oxidized peak at $700 \mathrm{~nm}$ stops growing after $\sim 2 \mathrm{~h}$ (around the time that the oxidant is expected to be used up as shown in Figure 3). The peak at $500 \mathrm{~nm}$ continues to grow, showing that reduced PTTh continues to be produced, possibly by a different mechanism. However, it is worth noticing that the position of the $\sim 500-\mathrm{nm}$ peak is not changing during the period of polymerization, indicating that the conjugation length remains the same for all polymers polymerized independent of polymerization time. It is also remarkable that the amount of oxidized PTTh remains unchanged and is not decreasing after $2 \mathrm{~h}$ of polymerization, excluding the possibility of one oxidized part of the polymer driving further polymerization after the 


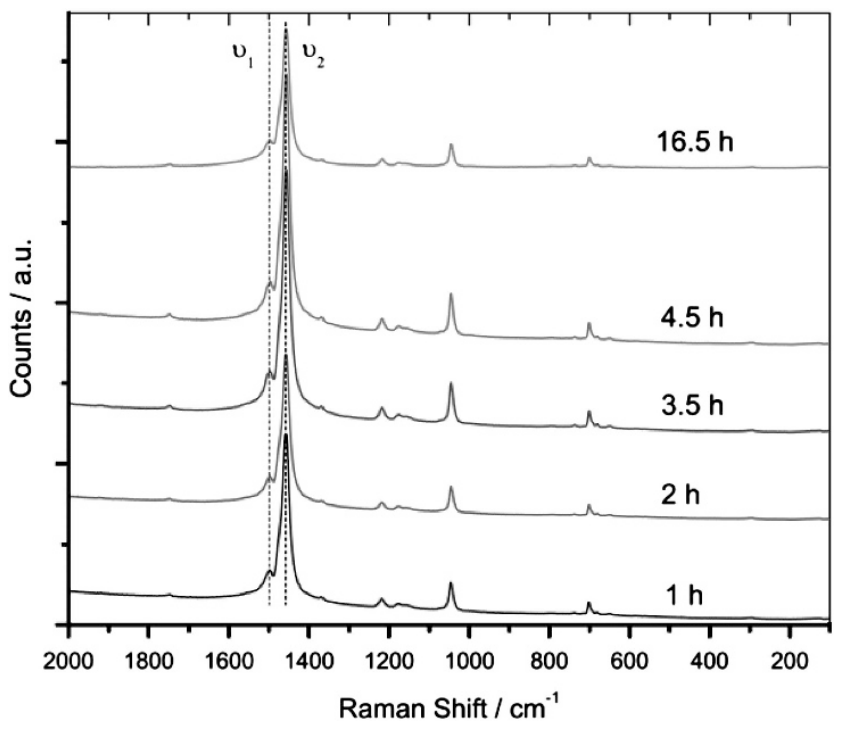

Figure 6 Raman spectra of PTTh with varying VPP time.

Fe(III) source of oxidant has been used up. The inset in Figure 5 shows the PTTh polymerized for $22 \mathrm{~h}$ after wash in ethanol. The polaronic band around $700 \mathrm{~nm}$ has disappeared, leaving a reduced form of the material.

Figure 6 displays the Raman spectra of the samples polymerized for various times after wash. All spectra show the features of polythiophene in the reduced state ${ }^{18}$ and, typical of the Raman spectra of polyconjugated materials, the relatively sparse bands originate from totally symmetric modes. ${ }^{19,20}$ The spectra are dominated by the bands in the $1500-1450 \mathrm{~cm}^{-1}$ region owing to the strong enhancement caused by electron-phonon coupling. ${ }^{19}$ The $v_{1}$ mode indicated in Figure 6 appears at $\sim 1500 \mathrm{~cm}^{-1}$ and is associated with the ring deformation of the ends of the polymer chains, while the $v_{2}$ mode situated at $1455 \mathrm{~cm}^{-1}$ is associated with ring deformation in the central part of the polymer chain. ${ }^{10,21,22}$ Through the deconvolution of this region, the integrated area intensity ratio between the $v_{1}$ and $v_{2}$ modes provides an indication of the length of the polymer chains. ${ }^{11}$ Although an apparently slight increase in chain length is seen as the polymerization proceeds up to $4.5 \mathrm{~h}$, the difference is far too small to draw any conclusions and well within the experimental/statistical errors, and is therefore not included in the presented data.

Frequency dispersion in conjugated polymers is well known ${ }^{10}$ and it has been shown that the $v_{1}$ mode in polythiophene is sensitive to the conjugation length of the polymer chains. ${ }^{21,23}$ Increased conjugation causes enhanced electron-phonon coupling that causes the Raman frequency of the $v_{1}$ mode to shift to lower values. ${ }^{21}$ In the present study, however, there was no significant or systematic change in the $v_{1}$ mode, indicating that between the samples there is no real change in the average conjugation length as also noticed above in the UV-vis measurements. It is interesting to note that the 16.5-h sample exhibited more fluorescence than the shorter polymerization times and required a quadratic baseline correction for display in Figure 6.

Combining the results, a few hypotheses can be made: first, during initial VPP, oxidized PTTh is formed, which has a ordered structure owing to $\mathrm{Fe}$ (III)PTS templating. Second, this occurs with $\mathrm{Fe}(\mathrm{III})$ as the oxidation agent as per usual understanding according to equation (1). Last, with continued polymerization, when the $\mathrm{Fe}$ (III) oxidant is 'used up' according to equation (1), a reduced and nontemplated, less-ordered form of PTTh is formed.

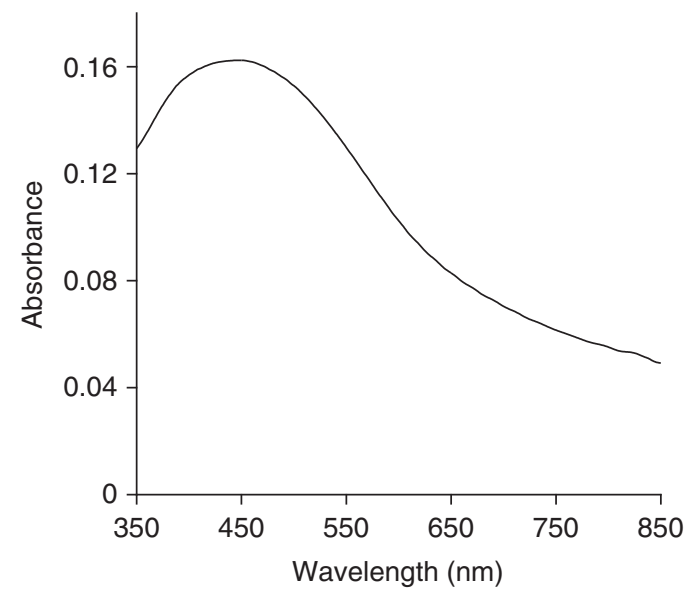

Figure 7 UV-vis spectra of VPP PTTh polymerized on glass with only PTSa as oxidant at $98^{\circ} \mathrm{C}$ for $6 \mathrm{~h}$.

The main observation that requires explanation in the present study remains that we are reaching a maximum polymerization point, where the polymerization stops-and that this point is about 2.3 times higher than one would expect from the common known polymerization route(s). It has earlier been shown that some thiophene derivatives can be polymerized by an acidic route to a nonconjugated form, that later can be converted to the conjugated form in a post-oxidation step. ${ }^{10}$ However, the UV-vis spectra clearly show that the reduced PTTh formed is conjugated and that the conjugation length remains unchanged with longer polymerization times (which is also confirmed with the Raman measurements), undermining this tempting explanation of an acidic polymerization route.

The unusual conditions used for VPP of TTh, $\sim 100{ }^{\circ} \mathrm{C}$ and no solvent (apart from liquid TTh), allow the possibility that some more 'exotic' reduction reactions could take place. It is worth reminding that the sulfur in the PTS actually is in a relatively high oxidation state and may have the potential to contribute to the TTh oxidation. In separate experiments, PTSa or poly(4styrenesulfonic acid) (PSS) was coated onto glass substrate from a $0.1 \mathrm{M}$ ethanol solution and then dried. PTSa and PSS films were then exposed to TTh vapor at $98^{\circ} \mathrm{C}$. Indeed coloration started to occur on the PTSa samples shortly after they were inserted into the VPP chamber, but no reaction could be detected on the PSS film. Figure 7 shows the UV-vis spectra of the PTSa-initiated film (6 $\mathrm{h}$ polymerization) after wash in ethanol, confirming that PTTh is formed.

PTSa is normally not considered an oxidant. However, desulsonation of PTSa at elevated temperatures is well known. The desulfonation of PTSa and other aromatic sulfonic acids was originally studied in detail by Wanders and Cerfontain, ${ }^{24,25}$ and was found to be a firstorder process with respect to the initial sulfonic acid concentration. Increase in temperature (over $100^{\circ} \mathrm{C}$ ) and acid concentration increased the rate constant of the desulfonation. The products of the desulfonation were confirmed to be toluene and $\mathrm{SO}_{3}$. This reversibility of the sulfonation process allows us to suggest that $\mathrm{SO}_{3}$ is formed during the VPP, after some of the initial Fe(III)PTS has been converted to Fe(II)PTS and PTSa (where the added proton is a product from the polymerization of TTh). 
Sulfuric acid and $\mathrm{SO}_{3}$, on the other hand, has been reported to be able to oxidize thiophenes at elevated temperatures, itself being reduced to $\mathrm{H}_{2} \mathrm{SO}_{3}$ or $\mathrm{SO}_{2}$, respectively. ${ }^{26,27}$ For $\mathrm{SO}_{3}$ and TTh:

$$
n \mathrm{SO}_{3}+n \mathrm{TTh} \Rightarrow n \mathrm{SO}_{2}+(\mathrm{PTTh})_{n}+n \mathrm{H}_{2} \mathrm{O}
$$

In the absence of aqueous conditions-as under VPP at $\sim 100{ }^{\circ} \mathrm{C}-\mathrm{SO}_{2}$ and $\mathrm{H}_{2} \mathrm{O}$ would then evaporate from the samples during VPP (equation (2)). Further, the gradually 'available' PTSa is converted to $\mathrm{SO}_{3}$, and $\mathrm{SO}_{3}$ is reduced to $\mathrm{SO}_{2}$ owing to the oxidation of TTh monomer. We are hereby suggesting the most likely scenario where the polymerization mechanism is maintained (as oxidative polymerization) but where a second oxidant than $\mathrm{Fe}(\mathrm{III})$ comes into play, namely the sulfur being reduced from oxidation state $6^{+}$to $4^{+}$.

QCM data for the PTTh polymerization with Fe(III)PTS were then considered as following. During the reduction of $\mathrm{Fe}(\mathrm{III})$ to $\mathrm{Fe}(\mathrm{II})$, one PTS per Fe(III) is 'released' from the iron complex; some of this is incorporated as counter ions in the doped PTTh formed during the 'use' of Fe(III) as oxidant (Figure 5). Normally a doping level of $\sim 0.25$ is reported for thiophenes. ${ }^{10,14}$ For TTh, this means that 8 (for the polymerization) +3 (for the oxidation) $\mathrm{Fe}$ (III) is needed to polymerize four units of TTh $\Rightarrow$ that $1 \mathrm{~mol}$ of $\mathrm{Fe}(\mathrm{III})$ would produce $0.36 \mathrm{~mol}$ of polymerized and oxidized TTh units containing $0.27 \mathrm{~mol}$ of PTS as dopant. This would then leave 0.73-mol PTS (that is not participating in the $\mathrm{Fe}(\mathrm{II})$ complex) per mole of initial $\mathrm{Fe}(\mathrm{III})$, that is now 'free' to form PTSa with a proton eliminated from TTh in the polymerization process. The possible amount of $\mathrm{SO}_{3}$ formed, according to the route suggested above, would then also be $0.73 \mathrm{~mol}$ per mole of initial $\mathrm{Fe}(\mathrm{III})$ and should so be able to polymerize a further $0.73 \mathrm{~mol}$ of (reduced) TTh units per mole of initial $\mathrm{Fe}(\mathrm{III})$, leading to a total of $(0.36+0.73)=1.09$ mole of TTh units per mole of initial Fe(III). This value is indeed close to values measured in the QCM experiments reported above (1:1.15).

These findings open a one-step polymerization route for VPP of thiophenes where the oxidant is not leaving any trace (apart from eventually as dopant) in the final product. This route is very attractive because it eliminates the possibility of iron contamination and saves a washing step to remove unwanted products from the conducting polymer material (that is, $\mathrm{Fe}(\mathrm{II})$ and excess of anions).

\section{CONCLUSIONS}

The polymerization of PTTh by VPP has been studied using a standard Fe(III)PTS oxidant and it was surprisingly found, when long polymerization times were used, that more than twice the amount of PTTh was produced compared with the expected amount according to the common understanding of the oxidative polymerization route using $\mathrm{Fe}(\mathrm{III})$ salts. The produced PTTh had similar conjugation length independent of polymerization time but showed less-ordered structure at longer polymerization times (more than $\sim 3 \mathrm{~h}$ ). The explanation for the large amount of PTTh polymerized was found to be owing to the PTS component of the oxidant and it is suggested that part of the PTS is converted to $\mathrm{SO}_{3}$ under VPP conditions and that the $\mathrm{SO}_{3}$ is capable of oxidizing the TTh monomer, itself being converted to $\mathrm{SO}_{2}$ and $\mathrm{H}_{2} \mathrm{O}$.

This finding is of importance as it is not only explaining the unexpected high yield of PTTh produced from more than $3 \mathrm{~h}$ polymerization but is also a discovery of an alternative oxidative polymerization route where the oxidant is converted to volatile compounds leaving no trace on the end product. Further development of this method will be reported in separate communications.

\section{ACKNOWLEDGEMENTS}

BWJ and OWJ gratefully acknowledge the Australian Research Council (ARC) for fellowships. ARC Centre of Excellent for Electromaterials Science is also acknowledged for OWJ research support. The authors acknowledge Dr Vanessa Armel for the ionic liquid.

1 Yanagida, S., Hanazawa, M., Kabumoto, A., Pac, C. \& Yoshino, K. Application of conducting polymers as catalysts for cis-trans photoisomerization of alkenes. Synth. Met. 18, 785-790 (1987).

2 Brydson, J. A. Plastics Materials. 7th edn (Butterworth-Heinemann, Great Britain, 1999).

3 Winther-Jensen, B., Winther-Jensen, O., Forsyth, M. \& MacFarlane, D. R. High rates of oxygen reduction over a vapor phase-polymerized PEDOT electrode. Science 321, 671-674 (2008)

4 Winther-Jensen, B., Fraser, K., Ong, C., Forsyth, M. \& MacFarlane, D. R. Conducting polymer composite materials for hydrogen generation. Adv. Mater. 22, 1727-1730 (2010).

5 Winther-Jensen, O., Desai, S., Shepherd, R. L., Innis, P. C., Winther-Jensen, B., Forsyth, M., Wallace, G. G. \& MacFarlane, D. R. Ion effects in REDOX cycling of conducting polymer based electrochromic materials. Electrochem. Commun. 12, 1505-1508 (2010).

6 Winther-Jensen, B. \& MacFarlane, D. R. New generation, metal-free electrocatalysts for fuel cells, solar cells and water splitting. Energy Environ. Sci. 4, 2790-2798 (2011).

7 Roncali, J. Conjugated poly(thiophenes): synthesis, functionalization, and applications. Chem. Rev. 92, 711-738 (1992).

8 Endo, K., Ogura, T., Hlgashihara, T. \& Ueda., M. A negative-type photosensitive poly(3hexylthiophene) with cross-linker and photoacid generator. Polym. J 41, 808-809 (2009).

9 Shimizu, H., Yamada, M., Wada, R. \& Okabe, M. Preparation and characterization of water self-dispersible poly(3-hexylthiophene) particles. Polym. J. 40, 33-36 (2008).

10 Skotheim, T. A., Elsenbaumer, R. L. \& Reynolds, J. R. Handbook of Conducting Polymers (Marcel Dekker, New York, 1998).

11 Bayley, P. M., Winther-Jensen, B., MacFarlane, D. R., Rocher, N. M. \& Forsyth, M. Enhanced properties in chemically polymerized poly(terthiophene) using vapour phase techniques. React. Funct. Polym. 68, 1119-1126 (2008).

12 Winther-Jensen, B., Chen, J., West, K. \& Wallace, G. Vapor phase polymerization of pyrrole and thiophene using iron(III) sulfonates as oxidizing agents. Macromolecules 37, 5930-5935 (2004).

13 Jeon, S. S., Yang, S. J., Lee, K. J. \& Im, S. S. A facile and rapid synthesis of unsubstituted polythiophene with high electrical conductivity using binary organic solvents. Polymer 51, 4069-4076 (2010).

14 Aasmundtveit, K. E., Samuelsen, E. J., Pettersson, L. A. A., Inganäs, O., Johansson, T. \& Feidenhans'l, R. Structure of thin films of poly(3,4-ethylenedioxythiophene). Synth. Met. 101, 561-564 (1999).

15 Stanford Research Systems QCM200 Operation and Service Manual (Stanford Research Systems, Inc., California, 2004).

16 Winther-Jensen, B., Forsyth, M., West, K., Andreasen, J. W., Bayley, P., Pas, S. \& MacFarlane, D. R. Order-disorder transitions in poly(3,4-ethylenedioxythiophene). Polymer 49, 481-487 (2008).

17 Zhang, D., Qin, J. \& Xue, G. Investigation of the electropolymerization of terthiophene in boron fluoride-ethyl ether. Synth. Met. 100, 285-289 (1999).

$18 \mathrm{Vu}$, Q. T., Pavlik, M., Hebestreit, N., Rammelt, U., Plieth, W. \& Pfleger, J. Nanocomposites based on titanium dioxide and polythiophene: structure and properties. React. Funct. Polym. 65, 69-77 (2005).

19 Agosti, E., Rivola, M., Hernandez, V., Del Zoppo, M. \& Zerbi, G. Electronic and dynamical effects from the unusual features of the Raman spectra of oligo and polythiophenes. Synth. Met. 100, 101-112 (1999).

20 Castiglioni, C., Del Zoppo, M. \& Zerbi, G. Vibrational Raman spectroscopy of polyconjugated organic oligomers and polymers. J. Raman Spectrosc. 24, 485-494 (1993).

21 Lopez Navarrete, J. T. \& Zerbi, G. Lattice dynamics and vibrational spectra of polythiophene. II: effective coordinate theory, doping induced, and photoexcited spectra. J. Chem. Phys. 94, 965-970 (1991).

22 Lopez Navarrete, J. T. \& Zerbi, G. Lattice dynamics and vibrational spectra of polythiophene. I: oligomers and polymer. J. Chem. Phys. 94, 957-964 (1991).

23 Geisselbrecht, J., Kurti, J. \& Kuzmany, H. Effective conjugation coordinate model: an investigation of polythiophene and polyisothianaphthene. Synth. Met. 57, 4266-4271 (1993).

24 Cerfontain, H. Mechanistic Aspects in Aromatic Sulfonution and Desulfination (Interscience, New York, 1968).

25 Wanders, A. C. M. \& Cerfontain, H. Kinetics of the desulfonation of benzenesulfonic acid and the toluenesulfonic acids in aqueous sulfuric acid. Recueil des Travaux Chimiques des Pays-Bas 86, 1199-1216 (1967).

26 Dai, W., Zhou, Y., Wang, S., Su, W., Sun, Y. \& Zhou, L. Desulfurization of transportation fuels targeting at removal of thiophene/benzothiophene. Fuel Process. Technol. 89, 749-755 (2008).

27 Nehlsen, J., Benziger, J. \& Kevrekidis, I. Oxidation of aliphatic and aromatic sulfides using sulfuric acid. Ind. Eng. Chem. Res. 45, 518-524 (2006). 